\section{Breast transillumination using the sinus diaphanograph}

Transillumination in the diagnosis of breast disease was first used about 50 years ago. ${ }^{1-3}$ The sinus diaphanograph is a recent development that provides a light beam of variable intensity. When it is used at the lowest possible setting small lesions become capable of light interference and more subtle changes in tissue density can be visualised.

Diaphanoscopy is performed in a totally darkened room. Normal and there was a two-week delay before the films were returned. No extra information was gained from these photographs.

The non-invasive nature of diaphanoscopy is a great benefit, and trials of the procedure are under way in Sweden. The ability of diaphanoscopy to detect minimal, non-palpable cancer is, however, unproved and our impression is that it would not be this sensitive. Although the most sophisticated instrument yet developed for transillumination, the diaphanograph has no place in a routine clinic but may occasionally be helpful in difficult cases, possibly reducing the number of referrals for mammography, or when mammography is unavailable. Alternatively diaphanoscopy may be repeated without risk and used to observe an at-risk group, particularly young women.

Diaphanoscopic appearance at first consultation compared with final diagnosis in 176 patients

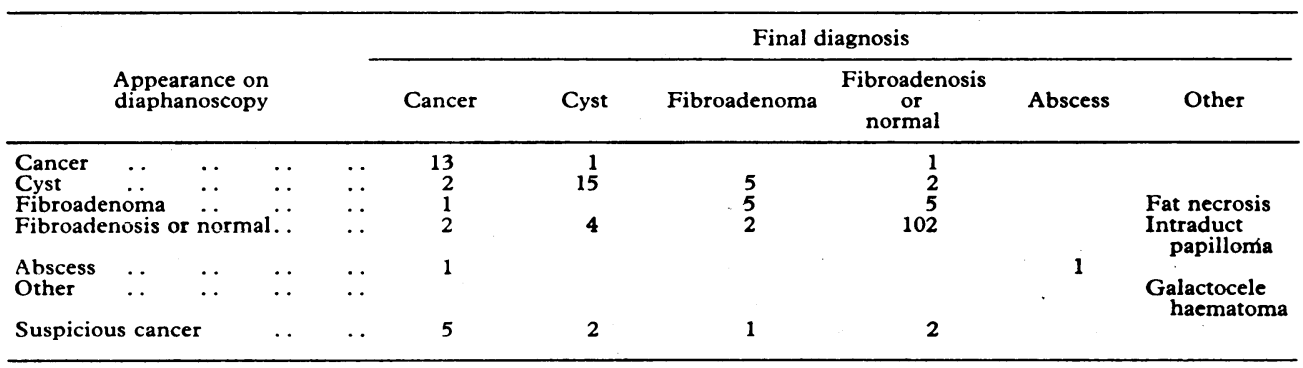

breast appears reddish yellow according to the amount of adipose tissue present, areas of increased density-that is, tumours-are darker than their surroundings, but cysts may appear light or dark depending on the fluid therein. The manufacturers suggest that differential diagnosis is improved by using infrared photography (diaphanography), and for this the light emitter has a built-in flash unit. Thus fibroadenomas appear red and malignant tissue dark brown or black.

\section{Patients, methods, and results}

Transillumination was performed on 176 patients (age range 16-80 years) without clinical information. The final diagnosis after biopsy or follow-up was made by an independent examiner. Diaphanoscopy took five minutes and diaphanography another five.

Seventy-eight lesions were detected by both diaphanoscopy and palpation and eight by palpation alone. Biopsy specimens were taken of these eight lesions, of which two were cancers. No impalpable lesions were detected by diaphanoscopy or mammography in this series. Overall, diaphanoscopy gave an accurate diagnosis in $143(81 \%)$ of the cases (table). By diaphanoscopy carcinoma was diagnosed or suspected in 18 of the 24 cases, and biopsy was recommended in $22(92 \%)$. Four cancers appeared benign on diaphanoscopy, and in two cases biopsy would not have been carried out on diaphanoscopy alone.

\section{Comment}

The object of the study was to see whether transillumination was of value as an aid to diagnosis and the need for biopsy. Biopsy (or aspiration) was recommended by diaphanoscopy in 65 cases, of which 55 showed appreciable disease and 10 were finally classified as fibroadenosis. Two cancers were missed: one was too high posteriorly to be successfully transilluminated, and the other was also missed on mammography. It was apparent that diaphanoscopy could not be used as the sole arbitrator for biopsy, and palpation proved to be more important.

Diaphanoscopy confirmed the clinical diagnosis of fibroadenosis in 102 out of 112 cases $(91 \%)$. Only in three cases of fibroadenosis $(2.7 \%)$ was biopsy recommended by transillumination because of a suspicion of carcinoma. Diaphanoscopy may be of value when there is clinical doubt about the diagnosis of fibroadenosis but is not necessary in most cases. It might, however, be repeated in young women with fibroadenosis to guard against the possibility of carcinoma, when repeated mammography may be inadvisable.

In difficult cases diaphanoscopy might prove a useful additional tool. Physical difficulties were experienced when examining lesions in small breasts, in the tail, and close to the chest wall, when escaping light tended to blind the observer. Although the manufacturers claim that diaphanography can be performed by a single operator this proved to be awkward. Infrared processing is not readily available
${ }^{1}$ Adair FE. Sanguinous discharge from the nipple and its significance in relation to cancer of the breast. Ann Surg 1930;91:197-209.

2 Cutler $M$. Transillumination as an aid in the diagnosis of breast tumours. Surg Gynecol Obstet 1929;48:721-9.

3 Donnelly BA. Nipple discharge: its clinical and pathological significance. Ann Surg 1950;131:342-55.

(Accepted 13 May 1981)

Department of Surgery, City Hospital, Nottingham

HW HOLLIDAY, FRCS, surgical research fellow

R W BLAMEY, MD, FRCS, professor of surgical science

\section{Attitudes to malarial prophylaxis}

Between 1976 and 1980 inclusive 376 cases of malaria were notified in Scotland. This hospital admitted 108 patients with Plasmodium vivax, seven with $P$ falciparum, and one with $P$ ovale infection.

All patients with falciparum malaria had taken some form of prophylaxis, though only two "satisfactorily" (satisfactory being defined as regular ingestion of adequate doses of an antimalarial for the duration of the trip plus one month afterwards). Six of the seven patients were on home leave from working in Africa. The problem appeared to be irregular medication, or drug resistance, or both.

Of 71 patients with vivax infection available in Scotland for advice before travelling (that is, not immigrants or visitors), 49 had taken no medication while the remaining 22 had taken it unsatisfactorily. The problem appeared to be failure to think of prophylaxis or to consider it important. This prompted us to study attitudes to prophylaxis among travellers to North-west India and Pakistan, where most $(93 \%)$ of the vivax malaria was contracted.

\section{Patients, methods, and results}

Contacts were made through a supermarket specialising in Asian foods, the National Bank of Pakistan, and other groups with Asian connections. A questionnaire was completed for each respondent describing his or her most recent trip in the past five years, with emphasis on antimalarial precautions taken.

There were 45 respondents, who had been accompanied by 59 relatives, 39 of whom were children below 15 years of age. In every instance relatives took the same immunisation and antimalarial precautions as the respondents. Twenty-one families had been to India and 24 to Pakistan. Responses were broadly similar for both groups.

How do you catch malaria? - Thirty-three respondents $(73 \%)$ knew that malaria was caught from the mosquito. Two thought food and drink played a 